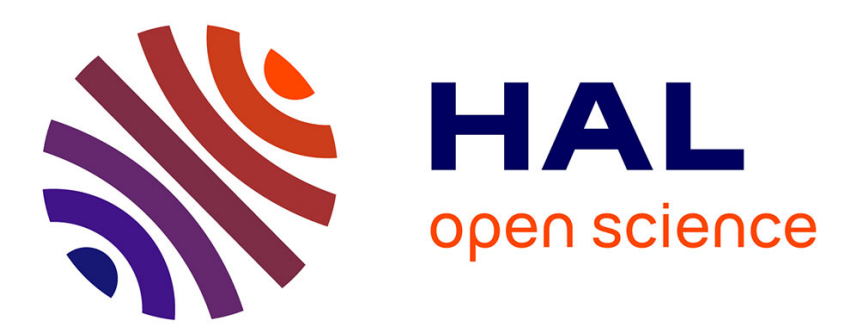

\title{
Segmentation of mesoscale ocean surface dynamics using satellite SST and SSH observations
}

Pierre Tandeo, Bertrand Chapron, Sileye Ba, Emmanuelle Autret, Ronan Fablet

\section{- To cite this version:}

Pierre Tandeo, Bertrand Chapron, Sileye Ba, Emmanuelle Autret, Ronan Fablet. Segmentation of mesoscale ocean surface dynamics using satellite SST and SSH observations. IEEE Transactions on Geoscience and Remote Sensing, 2013, 52 (7), pp.4227 - 4235. 10.1109/TGRS.2013.2280494 . hal00947965

\section{HAL Id: hal-00947965 \\ https://hal.science/hal-00947965}

Submitted on 19 Feb 2014

HAL is a multi-disciplinary open access archive for the deposit and dissemination of scientific research documents, whether they are published or not. The documents may come from teaching and research institutions in France or abroad, or from public or private research centers.
L'archive ouverte pluridisciplinaire HAL, est destinée au dépôt et à la diffusion de documents scientifiques de niveau recherche, publiés ou non, émanant des établissements d'enseignement et de recherche français ou étrangers, des laboratoires publics ou privés. 


\title{
Segmentation of mesoscale ocean surface dynamics using satellite SST and SSH observations
}

\author{
Pierre Tandeo*, Bertrand Chapron $^{\dagger}$, Sileye Ba* ${ }^{*}$ Emmanuelle Autret ${ }^{\dagger}$, Ronan Fablet* \\ *Institut Mines-Telecom, Telecom Bretagne, CNRS UMR 6285 LabSTICC, Pôle CID, Technopôle Brest Iroise, \\ 29238 Cedex, France \\ †IFREMER, Laboratoire d'océanographie spatiale, 29280 Plouzancé, France
}

\begin{abstract}
Multi-satellite measurements of altimeter-derived Sea Surface Height (SSH) and Sea Surface Temperature (SST) provide a wealth of information about ocean circulation, especially mesoscale ocean dynamics which may involve strong spatiotemporal relationships between SSH and SST fields. Within an observation-driven framework, we investigate the extent to which mesoscale ocean dynamics may be decomposed into a mixture of dynamical modes, characterized by different local regressions between SSH and SST fields. Formally, we develop a novel latent class regression model to identify dynamical modes from joint SSH and SST observation series. Applied to the highly dynamical Agulhas region, we demonstrate and discuss the geophysical relevance of the proposed mixture model to achieve a spatiotemporal segmentation of the upper ocean dynamics.
\end{abstract}

Index Terms-SST, SSH, Observation-driven model, Latent class regression, Mesoscale ocean surface dynamics

\section{INTRODUCTION}

$\mathbf{I}$ $\mathrm{N}$ the last two decades, multi-satellite measurements of altimeter-derived Sea Surface Height (SSH) and multisensor measurements of Sea Surface Temperature (SST) have provided a wealth of information about ocean circulation and atmosphere-ocean interactions. As a depth-integrated quantity dependent upon the density structure of the water column, altimeter SSH estimations capture mesoscale structures, horizontal scales of $50 \mathrm{~km}$ to few hundred kilometers, and allow for the retrieval of surface currents using the geostrophy balance. This emerging and rich mesoscale circulation further stirs the large-scale SST fields. Accordingly, our picture of upper ocean dynamics has considerably evolved towards a complex system characterized by strong interactions, whose spatio-temporal variability extends over a wide range of scales. Furthermore, several studies (cf. [20], [21], [17], [19], [13]) rationalize and demonstrate that fields of SST can become an active tracer coupled to the dynamics leading to strong correlations with SSH fields.

Such a framework can possibly guide the investigation and implementation of improved statistical means to optimally combine existing multi-altimeter SSH measurements with other satellite medium to high resolution observations (e.g., microwave sea surface temperature and salinity, scatterometer winds), augmented by the growing available in situ data (e.g., [1], [8], [26]). Theoretically, the upper ocean turbulence for the horizontal scales between $50 \mathrm{~km}$ to few hundred kilometers is still consistent with the geostrophy turbulence theory. Under this assumption, the upper ocean dynamics may be simply predicted from surface density horizontal variations possibly dominated by SST variations. For such a case, a linear transfer function shall be identified between SSH and SST fields to also possibly lead to the estimation of the subsurface flow (e.g., [16], [20]). This linear transfer function does not involve temporal differencing as in the maximum cross-correlation technique or alternate strategies (e.g., [4], [23], [7]). Note that other recent papers use nonlinear transfer functions to relate SST and SSH fields (e.g., [12]).

This strongly advocates for observation-driven studies to explore and characterize the local relationships between SST, SSH and the derived surface currents from satellite-based routine observations. Yet, as illustrated in Fig. 1, a simple linear transfer function cannot be expected to solely govern the whole mesoscale dynamics in a particular ocean region. As revealed, an overall spatial correlation exists, but for instance, relationships between SST gradients and altimetry-derived surface currents may spatially differ. In the warmer SST frontal zone, SST gradients correspond to large surface currents (top of the image). In the colder frontal area, large SST gradients do not reflect in large surface currents (bottom of the image). Besides, the clearly detected eddy (top-left of the image) is associated with weak SST gradients.

Within an observation-driven framework, one may consider joint PCA/EOF (Principal Component Analysis, Empirical Orthogonal Functions) procedures to decompose the relationships between SST and SSH fields, as widely used in ocean sensing applications (cf. [22], [6]). Such EOF-based schemes would however resort to a single linear and global model. As such, this model could not address the spatial non-stationarity of the SST-SSH relationships illustrated in Fig. 1. By contrast, we here consider local linear transfer functions. We assume that locally, upper ocean dynamics may be analyzed according to a finite mixture model, where each component of the mixture is characterized by a local SST-SSH linear transfer function. This mixture-based representation relates to a nonlinear model with assumptions in accordance with the observations made above between SST and surface currents. In this paper, we propose to investigate such a model to (i) develop a probabilistic learningbased setting for the inference of such mixture models and the spatio-temporal segmentation of the identified dynamical modes (i.e., the different components of the mixture model), and (ii) evaluate the extent to which such mixture models are geophysically relevant to characterize the upper ocean dynamics over active ocean regions. 
Hereafter we consider the Agulhas region, and the paper is organized as follows. Section II presents the remote sensing data and describes our probabilistic learning-based model. In Section III, the application to satellite observations is evaluated. We further discuss and summarize the key results of our investigations in Section IV.

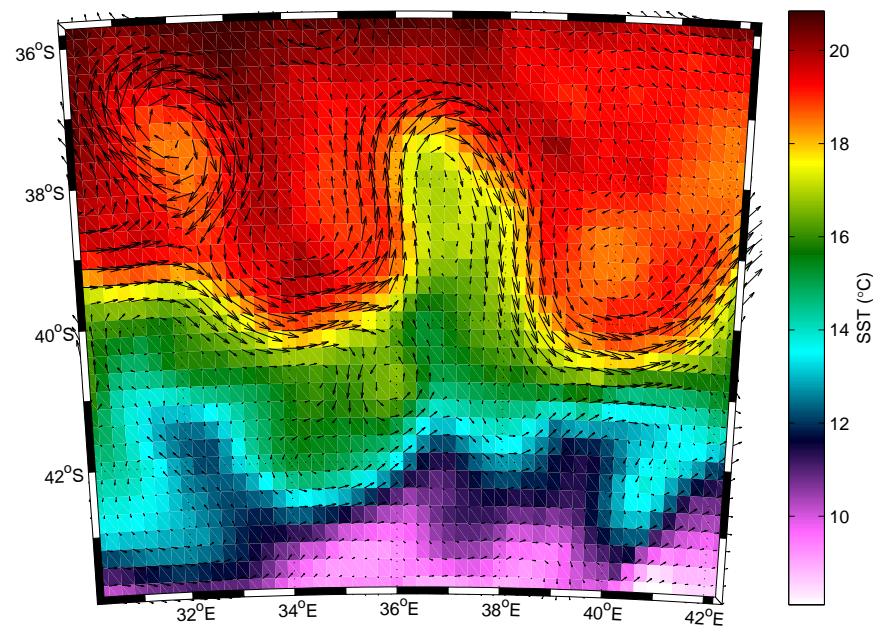

(a)

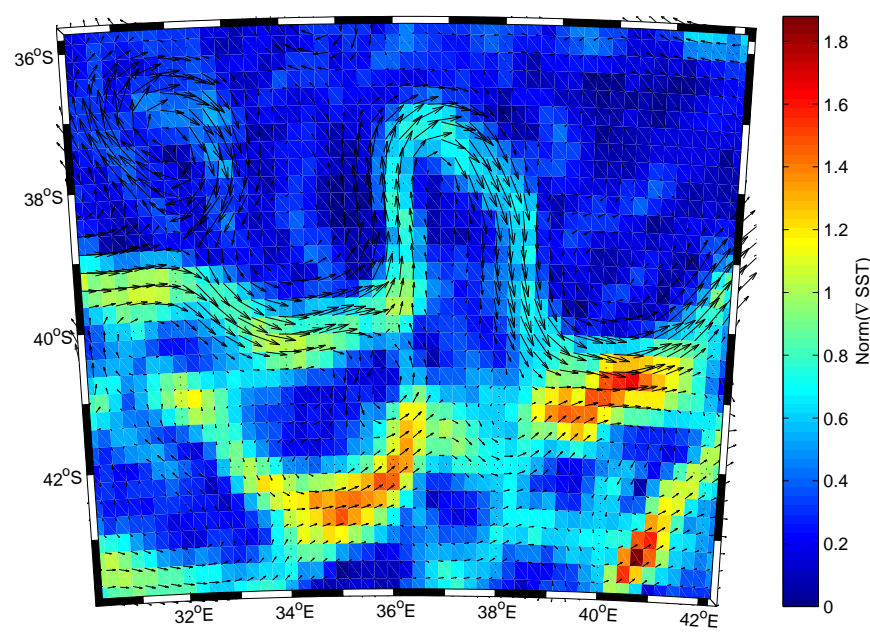

(b)

Fig. 1. Surface currents derived from altimeter SSHs and microwave SSTs (a) with the associated temperature gradient norms (b) within the Agulhas return current the $1^{\text {st }}$ of January, 2004.

\section{DATA AND METHODOLOGY}

\section{A. Patch-based approach}

As mentioned above, recent theoretical and numerical experiments have stressed that upper ocean dynamics may be characterized by couplings between SSH and SST according to the following relationship in the Fourier domain (cf. [14]):

$$
\mathcal{F}_{\mathcal{H}}(\widehat{\mathrm{SSH}})=-\gamma|\mathbf{k}|^{-\alpha} \mathcal{F}_{\mathcal{T}}(\widehat{\mathrm{SST}})
$$

where $\mathbf{k}$ is the horizontal wavenumber vector, $\mathcal{F}_{\mathcal{H}}$ and $\mathcal{F}_{\mathcal{T}}$ are linear filters of SSH and SST respectively. The $\alpha$ parameter sets up the effective coupling between surface fields. For $\alpha=1$, Eq. (1) resorts to the Surface Quasi-Geostrophy (SQG) model. In [16], $\mathcal{F}_{\mathcal{H}}$ and $\mathcal{F}_{\mathcal{T}}$ were band-pass filters between $80 \mathrm{~km}$ and $300 \mathrm{~km}$. As $\alpha$ increases, the smoothing increases and couplings decreases. For $\alpha=2$, the filtered SST would trace the vorticity. Formally, Eq. (1) states that surface currents can be regarded as spatial derivatives of a filtered version of the SST field. The parameter $\gamma$ relates to a normalization constraint. In general, parameters $\gamma$ and $\alpha$ as well as the definition of the filters $\mathcal{F}_{\mathcal{H}}$ and $\mathcal{F}_{\mathcal{T}}$, may spatially vary such that a single linear transfer function as in Eq. (1) is unlikely to apply globally as illustrated in Fig. 1.

These considerations led us to hypothesize that zonal and meridional surface currents noted $(\mathrm{U}, \mathrm{V})$ and $\mathrm{SSH}$ can still locally relate to SST derivatives, but according to a finite set of $K$ linear transfer functions, hereafter referred to as $K$ dynamical modes. Formally, this is stated in the Fourier domain as

$$
(\widehat{\mathrm{SSH}}, \widehat{\mathrm{U}}, \widehat{\mathrm{V}})=\mathcal{H}_{k}(\widehat{\mathrm{SST}})
$$

where $\mathcal{H}_{k}$ characterizes the $k^{t h}$ dynamical mode, which locally relates SSH and SST fields through linear filter $\mathcal{H}_{k}$. In this study, we do not consider any band-pass filters $\mathcal{F}_{\mathcal{H}}$ and $\mathcal{F}_{\mathcal{T}}$. Using a matrix formulation, Eq. (2) is rewritten in the real domain as a patch-based linear regression

$$
\mathbf{Y}\left(s_{i}, t_{i}\right)=\mathbf{H}_{k}\left(\mathbf{X}\left(s_{i}, t_{i}\right)\right)
$$

where $\mathbf{Y}\left(s_{i}, t_{i}\right)$ encodes the local $\mathrm{SSH}$ variability through a 3 -dimensional vector formed by the $\mathrm{SSH}$ value and the surface current $(\mathrm{U}, \mathrm{V})$ at spatio-temporal location $\left(s_{i}, t_{i}\right)$ and $\mathbf{X}\left(s_{i}, t_{i}\right)$ is the vectorized version of the local SST patch centered in $s_{i}$ at time $t_{i}$ (cf. Fig. 2). It may be noted that we encoded local $\mathrm{SSH}$ variations at spatio-temporal location $\left(s_{i}, t_{i}\right)$ through the surface currents which are computed as the spatial derivatives of the SSH field. As such, it constrains the method to account for spatial regularity. The linear operator associated with dynamical mode $k$ is corresponding to the local version of $\mathcal{H}_{k}$ in Eq. (2). It corresponds to three vectorized versions of spatial convolution matrices. Here, $p$ defines the size of the local SST neighborhood around $s_{i}$ and is set according to the Rossby radius of the study region, i.e. the mean size of the mesoscale ocean structures like eddies. For the Great Agulhas current region, we set it up to $200 \mathrm{~km}$, i.e. $p=81$ for the spatial resolution of the considered data.

\section{B. Remote sensing data}

As SSH and surface geostrophic current (U,V) data, we use the daily delayed time Maps of Absolute Dynamic Topography (MADT) produced by Collecte Localisation Satellites (CLS) available online at http://www.aviso.oceanobs.com/. This information combines the signal of several altimeters onto a $1 / 3^{\circ}$ Mercator projection grid. We use the 2004 data since four altimeters were available (Jason-1, Envisat or ERS-2, Topex/Poseidon and GFO). As SST data, we use optimally interpolated microwave SSTs provided by Remote Sensing System (RSS) available online at http://www.ssmi.com/. It combines the signal of three microwave radiometers (TMI, AMSR-E and WindSAT) which are robust to the presence of clouds. The spatial resolution is $1 / 4^{\circ} \times 1 / 4^{\circ}$ and the temporal 


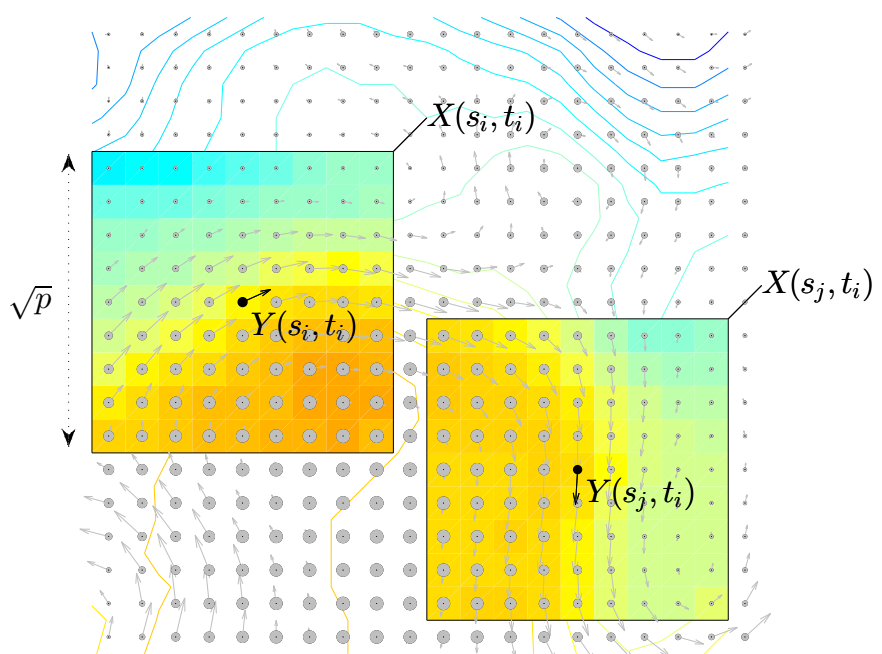

Fig. 2. Sketch of SST patches (in degree represented in false colours, the more red the hoter), noted $\mathbf{X}$, and the corresponding SSHs (in meter represented by dots, the bigger the higher) and surface currents (in meter per second represented by quivers, the longer the stronger) noted $\mathbf{Y}$ at the central location $s_{i}$ and $s_{j}$ at time $t_{i}$.

resolution is the same as the MADT data, i.e. daily. We bilinearly interpolate the MADT data onto the SST grid. We focus on the Agulhas region between longitudes $5^{\circ} \mathrm{E}$ to $65^{\circ} \mathrm{E}$ and latitudes $30^{\circ} \mathrm{S}$ to 48 degreeS.

Given the joint series of satellite observations, we extract SST patches (noted $\mathbf{X}$ ) and the associated SSH with surface current $(\mathrm{U}, \mathrm{V})$ at the center of the patches (noted $\mathbf{Y})$. Overall, the processed dataset is composed of $\sim 5 \times 10^{6}$ pairs of vectors $\mathbf{X}$ and $\mathbf{Y}$. To infer the parameters of the considered mixture model, i.e. the parameters of each dynamical mode in Eq. (3), we first build a training dataset as a random sample of $n=10^{5}$ elements (for a given day, we use about $2 \%$ of the data to fit the model). In a second step, we apply the inferred mixture model to the entire processed dataset to extract the spatio-temporal of the different dynamical modes.

\section{$C$. Latent class regression model}

Our objective is to identify $K$ different dynamical modes (latent variable $Z$ ) from a joint set of SST patches ( $p$ dimensional vector $\mathbf{X}$ ) and SSH with zonal and meridional surface currents (3-dimensional vector $\mathbf{Y}$ ). In this paper, we assume that the conditional probability of $\mathbf{Y}$ given $\mathbf{X}$ and the dynamical mode $Z=k$ is given by

$$
p(\mathbf{Y} \mid \mathbf{X}, Z=k) \propto \mathcal{N}_{k}\left(\mathbf{Y} ; \mathbf{X} \boldsymbol{\beta}_{k}, \boldsymbol{\Sigma}_{k}\right)
$$

where $\mathcal{N}_{k}$ represents a multivariate Gaussian probability density function evaluated in $\mathbf{Y}$ with mean $\mathbf{X} \boldsymbol{\beta}_{k}$ and covariance $\Sigma_{k}$. Hence, the conditional probability of $\mathbf{Y} \mid \mathbf{X}$ resorts to a mixture of Normal distributions

$$
p(\mathbf{Y} \mid \mathbf{X}, \boldsymbol{\theta})=\sum_{k=1}^{K} \lambda_{k} \mathcal{N}_{k}\left(\mathbf{Y} ; \mathbf{X} \boldsymbol{\beta}_{k}, \boldsymbol{\Sigma}_{k}\right)
$$

where $\lambda_{k}$ is the prior probability of mode $k$. To simplify the notations, we store the overall parameters of Eq. (5) in $\boldsymbol{\theta}=\left(\lambda_{1}, \ldots, \lambda_{K}, \boldsymbol{\beta}_{1}, \ldots, \boldsymbol{\beta}_{K}, \boldsymbol{\Sigma}_{1}, \ldots, \boldsymbol{\Sigma}_{K}\right)$. In the literature, this model is referred to as a "latent class regression" or "clusterwise regression" (cf. [10]). By construction, it imposes that $0 \leq \lambda_{k} \leq 1, \sum_{k=1}^{K} \lambda_{k}=1$ and $\boldsymbol{\Sigma}_{k}$ is positive defined. The maximum likelihood estimation procedure for model parameters $\boldsymbol{\theta}$ is given below.

\section{Model learning}

To learn model parameters $\boldsymbol{\theta}$ in Eq. (5), we resort to a maximum likelihood criterion and use an iterative ExpectationMaximization (EM) procedure (cf. [9]). It relies on the maximization of the log-likelihood given by

$$
\mathcal{L}(\boldsymbol{\theta})=\sum_{i=1}^{n} \log \left(p\left(\mathbf{Y}\left(s_{i}, t_{i}\right) \mid \mathbf{X}\left(s_{i}, t_{i}\right), \boldsymbol{\theta}\right)\right)
$$

where $n$ is the number of observations of the training dataset described in Section II-B. From a given initialization, the EM procedure iterates an E-step (Expectation-step) and M-step (Maximization-step). At a given iteration, using the Bayes theorem, the E-step resorts to the computation of the posterior probabilities of the latent variable $Z$ for each spatio-temporal location $\left(s_{i}, t_{i}\right)$ given current parameter estimate $\hat{\boldsymbol{\theta}}$ :

$$
\hat{\pi}_{k}\left(s_{i}, t_{i}\right)=\frac{\hat{\lambda}_{k} \mathcal{N}_{k}\left(\mathbf{Y}\left(s_{i}, t_{i}\right) ; \mathbf{X}\left(s_{i}, t_{i}\right) \hat{\boldsymbol{\beta}}_{k}, \hat{\boldsymbol{\Sigma}}_{k}\right)}{p\left(\mathbf{Y}\left(s_{i}, t_{i}\right) \mid \mathbf{X}\left(s_{i}, t_{i}\right), \hat{\boldsymbol{\theta}}\right)}, \forall k .
$$

The M-step then minimizes the expectation of the $\log$ likelihood conditionally to the current parameter estimate $\hat{\boldsymbol{\theta}}$. This leads to the update of the prior probabilities of the latent variable $Z$ as

$$
\hat{\lambda}_{k}=\frac{\sum_{i=1}^{n} \hat{\pi}_{k}\left(s_{i}, t_{i}\right)}{n}, \forall k .
$$

The updated regression parameters $\hat{\boldsymbol{\beta}}_{k}, \forall k$ are derived by fitting $K$ distinct linear regressions using a weighted least square criterion on the $n$ observations where the weights are given by the posterior probabilities given in Eq. (7) as in [25]. Then, we maximize $\mathcal{L}$ with respect to $\boldsymbol{\Sigma}_{k}$ and obtain

$$
\hat{\boldsymbol{\Sigma}}_{k}=\frac{\sum_{i=1}^{n} \hat{\pi}_{k}\left(s_{i}, t_{i}\right) \boldsymbol{\epsilon}_{k}\left(s_{i}, t_{i}\right)^{\top} \boldsymbol{\epsilon}_{k}\left(s_{i}, t_{i}\right)}{\sum_{i=1}^{n} \hat{\pi}_{k}\left(s_{i}, t_{i}\right)}, \forall k
$$

where $\boldsymbol{\epsilon}_{k}\left(s_{i}, t_{i}\right)=\mathbf{Y}\left(s_{i}, t_{i}\right)-\mathbf{X}\left(s_{i}, t_{i}\right) \hat{\boldsymbol{\beta}}_{k}$ and ${ }^{\top}$ represents the transpose. The algorithm iterates the E-step and M-step until a negligible increase of the $\log$-likelihood $\mathcal{L}$ which is strictly growing.

A key aspect of the latent class regression is the choice of $K$, the number of clusters. Different statistical criteria state the selection of parameter $K$ as a trade-off between the likelihood and the complexity of the model (cf. [18]) such as the Akaike Information Criterion (AIC). However, the optimization of these criteria makes no effort to distinguish the error explained by the regression fit and the error explained by the clustering process. In practice, there is an actual potential for overfitting with latent class regression model (cf. [5]). Then, for a given number of clusters $K$, we suggest different evaluations of the EM algorithm to reach a greater consistency in the estimation of model parameters (cf. [15]). The idea is 
to use random values $\hat{\pi}_{k}$ as initialization values of the EM procedure and select parameter estimates corresponding to the greatest likelihood (see [3] for more details). In our case, the inference based on AIC would lead to $\mathrm{K}$ values between 4 and 9 . We performed a complementary qualitative analysis and the setting $K=4$ resulted as a good trade-off between the geophysical interpretation of the model and regression error statistics (i.e., the maximization of the likelihood).

\section{E. Spatio-temporal segmentation of dynamical modes and SSH/current predictions}

We exploit the inferred mixture model with parameter $\hat{\boldsymbol{\theta}}$ to perform a spatio-temporal segmentation of the underlying dynamical modes. More precisely, for any spatial location $s$ and time $t$ within the Agulhas region over the year 2004 (cf. processed data in Section II-B), we use Eq. (7) to evaluate the posterior probability $\hat{\pi}_{k}(s, t)$ for the $K=4$ dynamical modes. Then, the pixels will be assigned to the most likely dynamical mode. One can also estimate for each time $t$, the relative spatial occurrence of each dynamical mode using Eq. (8). Besides, using Eq. (3), the estimation of the SSH and surface current at the spatial location $s$ and time $t$ from the associated SST patch follows from the fuzzy regression

$$
\hat{\mathbf{Y}}(s, t)=\sum_{k=1}^{K} \hat{\pi}_{k}(s, t) \mathbf{X}(s, t) \hat{\boldsymbol{\beta}}_{k}
$$

where the $\hat{\boldsymbol{\beta}}_{k}$ parameterize the linear operator $\mathbf{H}_{k}$. They correspond to the $p \times 3$ regression coefficient matrices between the patch of SSTs ( $\mathbf{X}$ in degree) and the central value of $\mathrm{SSH}$ along with zonal $\mathrm{U}$ and meridional $\mathrm{V}$ components of the surface currents ( $\mathbf{Y}$ in meter and meter per second). All the locations of the Agulhas current share the same $K$ matrices $\hat{\boldsymbol{\beta}}_{k}$ which are not changing temporally nor spatially. It may be outline that no additional constraint is set on the posterior probabilities $\hat{\pi}_{k}(s, t)$, which could reveal space-time variations of the distribution of the dynamical modes (including seasonal variations).

\section{CHARACTERIZATION OF OCEAN SURFACE DYNAMICS}

We first report the temporal evolution of the relative spatial occurrence of the $K=4$ dynamical modes (cf. Fig. 3). The dynamical modes involve clear seasonal cycles. Dynamical modes 1 (red) and 4 (blue) depict similar temporal variations, completely out of phase with dynamical modes 2 (green) and 3 (cyan). To study the spatial distribution of these dynamical modes, we focus on two dates corresponding to the maximal and minimal values of the seasonal cycle, namely the $1^{\text {st }}$ of March and the $1^{\text {st }}$ of September, 2004. They correspond to the maximum or minimum values of the $\hat{\lambda}_{k}$.

For these two dates, from the maps of posterior probabilities $\hat{\pi}_{k}(s, t)$, we determine the segmentation maps of each dynamical mode as illustrated in Fig. 4. The animations of the time series of these daily maps in the Agulhas current over 2004 are available as supplementary material or online at: http://tandeo.wordpress.com/communications/articles/. A first qualitative analysis of these maps highlights a clear spatiotemporal segmentation of the different dynamical modes that

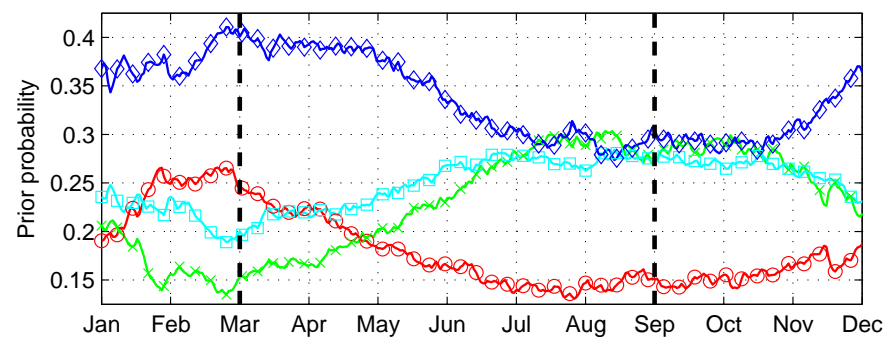

Fig. 3. Time series of the relative proportion of locations associated with the four dynamical modes within the Agulhas region for the year 2004. In the subsequent, colors red, green, cyan and blue respectively distinguish the first, second, third and fourth dynamical modes. The straight lines correspond to a six month time separation: $1^{\text {st }}$ of March and $1^{\text {st }}$ of September, 2004. The corresponding spatial maps of the posterior probabilities for each dynamical mode are given in Fig. 4.

can be interpreted from a geophysical perspective in terms of different geophysical processes. We also report for each dynamical mode the observed distributions of current, height and temperature values (cf. Fig. 5). The first dynamical mode (red) characterizes very strong current magnitude and warm waters. It is primarily associated with the main Agulhas current that flows down the East coast of Africa through the Agulhas ridge. This mode also involves mesoscale eddies, the so-called warm core Agulhas rings with strong surface currents, low temperature gradients and middle-range $\mathrm{SSH}$ values around $0.5 \mathrm{~m}$. The later seems to be a discriminative feature of this first mode. The second dynamical mode (green) mainly relates to the eastward Agulhas return current that hits a part of the South Atlantic current. It creates a subtropical front varying from $36^{\circ} \mathrm{S}$ to $44^{\circ} \mathrm{S}$ with strong eastward currents, middlerange SST gradients and large SSH values (about $1 \mathrm{~m}$ ). This front was clearly observed in the upper part of Fig. 1(b). The third (cyan) and fourth (blue) dynamical modes correspond to weaker surface currents. The third one is characterized by mid-temperatures and westward currents whereas the fourth one involves colder temperatures and eastward currents. Let us stress that the third dynamical mode involves large SST gradients but weak surface currents as identified in the lower part of Fig. 1(b). In this mode, the SST can be clearly identified as a passive tracer of the surface upper ocean dynamics.

To characterize more precisely the inferred model, we plot the regression line and the $95 \%$ confidence region from the estimated parameters $\hat{\boldsymbol{\beta}}_{k}$ and $\hat{\boldsymbol{\Sigma}}_{k}$ of each dynamical mode (cf. Fig. 6). These results clearly stress the relevance of a mixture model, compared to a single linear model (represented as the black line). Similar slopes are observed for the second and third (green and cyan) dynamical modes as well as the first and fourth (red and blue) ones. Stronger differences among the dynamical modes are outlined regarding the associated regression error variances. We can notice that the the first mode involves the greater variance and the fourth one the lower one.

We further investigate the geophysical consistency of the identified dynamical modes from the comparison between the true MADT SSH and surface currents in Fig. 7(a) and the 


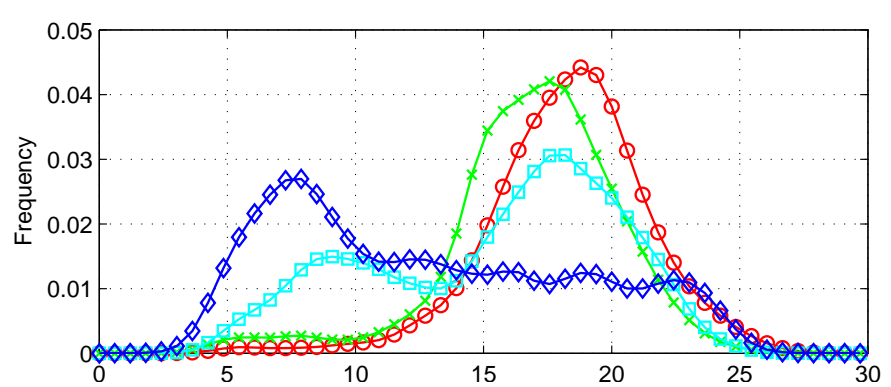

(a) $\operatorname{SST}\left({ }^{\circ} \mathrm{C}\right)$

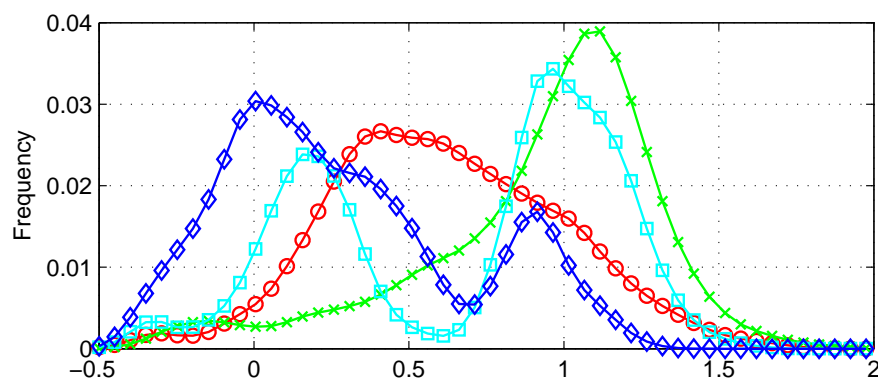

(b) SSH (m)

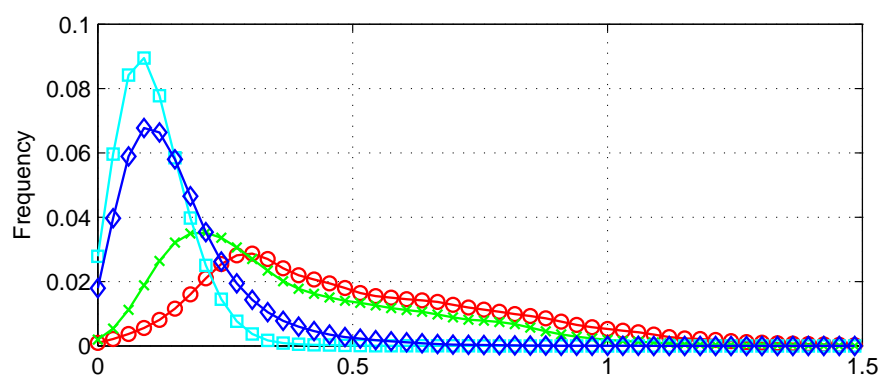

(c) $\operatorname{Norm}(\mathrm{U}, \mathrm{V})(\mathrm{m} / \mathrm{s})$
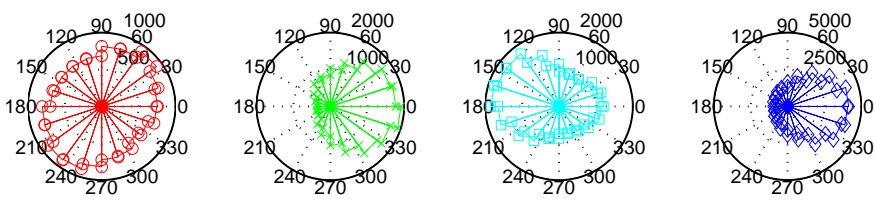

(d) Angle(U,V)

Fig. 5. Distribution of the SST (a), SSH (b), surface current (U,V) norm (c) and direction (d). The results are given for each dynamical mode within the Agulhas current for the whole year 2004.

prediction of the latent class regression model in Fig. 7(b) given by Eq. (10). Overall, as reported in Table I, a good agreement is obtained with a global correlation coefficient of 0.72 for the surface currents and 0.96 for the $\mathrm{SSH}$; it can locally be very large as illustrated in the right column of Fig. 7 (corresponding to the zone depicted in Fig. 1). This zone involves the four dynamical modes. The mixture model enables us to retrieve both the large warm eddy associated with weak SST gradients (top-left of the zone and first dynamical mode), the relatively large surface currents along the large warmer SST gradients (upper part and second mode), as well as the rather weak currents along the large but colder SST front (lower part and third mode). For comparison purposes, we also plot the results issued from a single linear transfer function in Fig. 7(c). This model clearly underestimates the surface
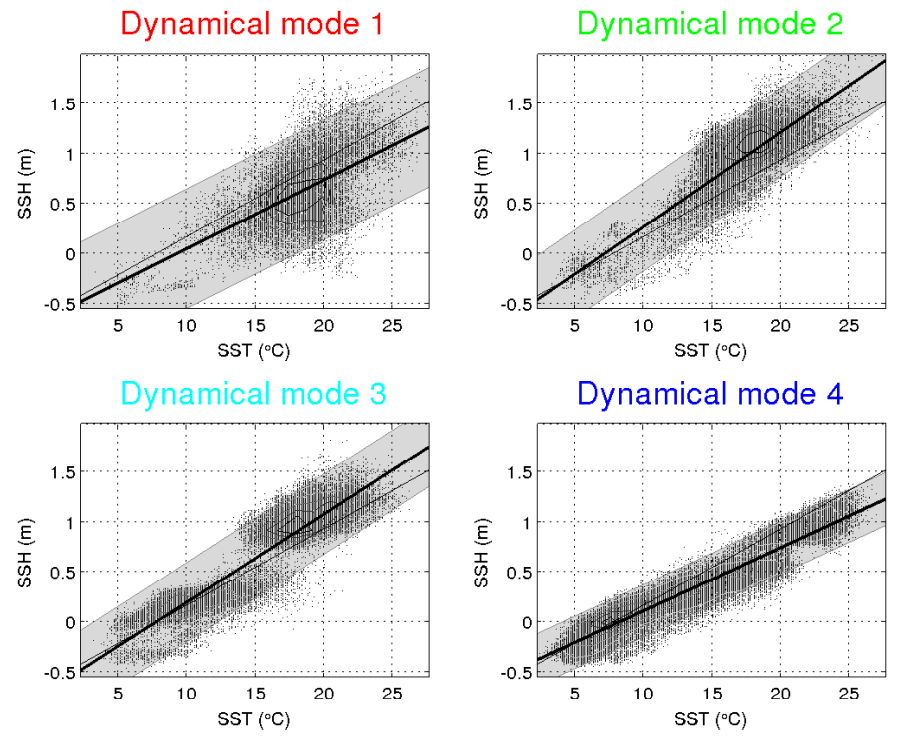

Fig. 6. SSH as a function of SST. For each dynamical mode, we give the regression line and the $95 \%$ confidence envelope. The four regressions are highly significant ( $\mathrm{p}$-values $<10^{-3}$ ). The black line is a benchmark curve corresponding to the global linear regression (with a one-mode model).

currents within the warm eddy (top-left) and overestimates the currents of the colder frontal zone (lower part), which stresses the requirement for considering a mixture model.

To further characterize each dynamical mode, we report in Table II the correlation and RMSE statistics computed within the associated spatio-temporal domain, i.e. the domain comprising all spatio-temporal locations assigned to dynamical mode $k$ according to posterior probabilities $\hat{\pi}_{k}(s, t)$ computed in Eq. (7). We compare the latent class regression model with respect to both the true MADT data and a SQG-like hypothesis, i.e. Eq. (1) with $\alpha=1$, within the space-time region associated with each dynamical mode. This analysis clearly discriminates the second and fourth modes from the first and third modes. The linear transfer functions of the second and fourth dynamical modes capture a large part of the variability of the true SSH data. These first two modes have also a good consistency with the SQG hypothesis, correlation coefficients of 0.63 and 0.68 , respectively. These results suggest that the SST might be regarded as an active tracer of the surface dynamics in the associated regions. By contrast, the SQG hypothesis poorly fits to the first and third dynamical modes, with correlation coefficients of 0.33 and 0.27 , respectively. These two dynamical modes also involve a slightly lower predictability of the linear transfer functions to retrieve the SSH and surface currents.

To summarize, the four dynamical modes correspond to different physical parameter values in Eq. (1). The second and fourth modes seem to correspond to $\alpha \simeq 1$ (close to the SQG model) whereas the first and fourth modes appear to be $\alpha>1$ (coupling of SST and SSH at large scales). The factor $\gamma$ relates to the amplitude of the surface geostrophic currents: large values relate to strong currents as retrieved for modes 1 and 2, and low values relate to strong currents as observed for modes 3 and 4. Overall, these results are consistent with the previous 
work reported in [16] and [27]. In particular, Isern-Fontanet et al. explored the SQG hypothesis from a phase-correlation analysis between SSH and SST fields, while Xu et al. explored the coupling assumption from a spectral analysis of $\mathrm{SSH}$ fields. These authors concluded that SQG-like dynamics would mainly occur near the edge of the large current system. Compared to these analysis, our contribution is two-fold: the quantitative characterization of the extent to which the SQG dynamics applies through correlation statistics as well as the actual space-time tracking of the regions associated with SQGlike and non-SQG dynamical modes.

\section{CONCLUSION AND PERSPECTIVES}

In this paper, we propose an observation-driven framework to identify, characterize and track ocean surface dynamical modes. We rely on a latent class regression model, where the dynamical modes are characterized by a local linear transfer function between SST, SSH and surface current $(\mathrm{U}, \mathrm{V})$, in agreement with the theoretical assumption given in Eq. (1). This probabilistic approach locally relates the distribution of the SSH and sea surface currents conditionally to the SST via a nonlinear model: a Gaussian mixture of linear transfer functions. The statistical parameters of the model are estimated using a maximum likelihood approach.

We applied the proposed methodology to the 2004 daily $1 / 4^{\circ} \times 1 / 4^{\circ}$ satellite SST and SSH image series. The reported results retrieved a relevant spatio-temporal decomposition of ocean surface dynamics in the Agulhas region according to four dynamical modes: (i) the main Agulhas current and warm core rings characterized by strong currents and hot temperatures where the SST is weakly correlated with SSH, (ii) the return Agulhas current with lower temperatures and currents where the SST is an active tracer, (iii) local front regions where strong SST gradients do not seem to affect the current velocities, and (iv) a weaker dynamical mode where SST is strongly correlated to SSH.

Our study complements previous theoretical studies which showed that mesoscale upper ocean dynamics may be characterized by a linear coupling between SST and SSH (cf. [20], [21], [17], [19], [13]). Following a fully observation-driven framework, the proposed latent regression model enabled us to identify different dynamical modes, including some SQGlike ones, and to track the space-time extension of each dynamical mode. The reported results clearly pointed out the requirement for considering a mixture model to decompose the space-time variabilities of the ocean surface dynamics. Regarding methodological aspects, it may be pointed out that EOF-based schemes (cf. [22], [6]) could not reveal such nonstationary space-time variabilities. Joint EOF scheme typically decomposes a global linear mapping between the analyzed fields according to principal modes. In our case, such an EOF decomposition could be considered for each dynamical mode to further characterize the associated linear transfer function with respect to joint SST-SSH principal modes.

Regarding our future work, we will further investigate latent class regression models with additional regressors. Among others, it seems appealing to explore how time-lagged SST features and other geophysical fields such as wind speed, mixed-layer depth, salinity and chlorophyll-a concentration (cf. [24]) could improve the estimation of SSH and surface currents. We also plan to apply the proposed model to other strongly active ocean regions such as the Gulf Stream system. Our objective will be to determine shared and/or systemspecific dynamical modes. Future work will also investigate more detailed physical interpretation of the identified dynamical modes, especially in terms of spectral characteristics. For instance, it would be interesting to relate more precisely the physical parameters $\gamma$ and $\alpha$ of Eq. (1) to the different hidden dynamical modes extracted by our statistical approach. Whereas factor $\gamma$ seems to be well estimated in this present paper, the spatial resolution of the satellite data (up to $50 \mathrm{~km}$ ) does not permit to detect dynamical modes with $\alpha<1$, corresponding to more local couplings between geophysical fields. Besides, such an improved model shall then possibly address both (i) the higher resolution prediction of mesoscale ocean surface currents from SST spatio-temporal fields (and improve the non geostrophic components estimation as in [23], [7]), and (ii) the extraction of new local and global descriptors of ocean surface dynamics from satellite sea surface observations (cf. [2], [11]).

\section{ACKNOWLEDGMENTS}

We would like to thank the Archiving, Validation and Interpretation of Satellite Oceanographic (AVISO) and the RSS projects for respectively providing the altimeter-derived SSH and surface current, and the microwave SST data. This study was partially supported by grant CREATE FI2O, the LabexMER, ANR RedHots and Institut Mines-Telecom. We are grateful to the reviewers and the editor for their numerous constructive comments.

\section{REFERENCES}

[1] M. M. Ali, D. Swain and R. A. Weller, "Estimation of ocean subsurface thermal structure from surface parameters: A neural network approach," Geophysical Research Letter, vol. 31, pp. L20308, 2004.

[2] S. O. Ba, E. Autret, B. Chapron and R. Fablet, "Statistical descriptors of ocean regimes from the geometric regularity of SST observations," Geoscience and Remote Sensing Letters, IEEE, vol. 9, pp. 851-855, 2012.

[3] C. Biernacki, G. Celeux and G. Govaert, "Choosing starting values for the EM algorithm for getting the highest likelihood in multivariate gaussian mixture models," Computational Statistics \& Data Analysis, vol. 41, pp. 561-575, 2003.

[4] M. M. Bowen, W. J. Emery, J. L. Wilkin, P. C. Tildesley, I. J. Barton and R. Knewtson, "Extracting multiyear surface currents from sequential thermal imagery using the maximum cross-correlation technique," Journal of Atmospheric and Oceanic Technology, vol. 19, pp. 1665-1676, 2002.

[5] M. J. Brusco, J. D. Cradit, D. Steinley and G. L. Fox, "Cautionary remarks on the use of clusterwise regression," Multivariate Behavioral Research, vol. 43, pp. 29-49, 2008.

[6] K. S. Casey and D. Adamec, "Sea surface temperature and sea surface height variability in the North Pacific Ocean from 1993 to 1999," Journal of Geophysical Research, vol. 107, 2002.

[7] W. Chen, "Surface velocity estimation from satellite imagery using displaced frame central difference equation," Geoscience and Remote Sensing, IEEE Transactions on, vol. 50, pp. 2791-2801, 2012.

[8] P. C. Chu, C. Fan and W. T. Liu, "Determination of vertical thermal structure from sea surface temperature," Journal of Atmospheric and Oceanic Technology, vol. 17, pp. 971-979, 2000.

[9] A. P. Dempster, N. M. Laird and D. B. Rubin, "Maximum likelihood from incomplete data via the EM algorithm," Journal of the Royal Statistical Society. Series B (Methodological), vol. 39, pp. 1-38, 1977. 
[10] W. S. DeSarbo and W. L. Cron, "A maximum likelihood methodology for clusterwise linear regression," Journal of Classification, vol. 5, pp. 249-282, 1988

[11] R. Fablet, A. Chaigneau and S. Bertrand, "Multiscale geometric deformations along planar curves: application to satellite tracking and ocean observation data," Geoscience and Remote Sensing, IEEE Transactions on, to appear.

[12] L. Gaultier, J. Verron, J. M. Brankart, O. Titaud and P. Brasseur, "On the inversion of submesoscale tracer fields to estimate the surface ocean circulation," Journal of Marine Systems, in press.

[13] U. Hausmann and A. Czaja, "The observed signature of mesoscale eddies in sea surface temperature and the associated heat transport," Deep Sea Research Part I: Oceanographic Research Papers, vol. 70, pp. 60-72, 2012.

[14] I. M. Held, R. T. Pierrehumbert, S. T. Garner and K. L. Swanson, "Surface quasi-geostrophic dynamics," Journal of Fluid Mechanics, vol. 282, pp. 1-20, 1995.

[15] C. Hennig, "Identifiablity of models for clusterwise linear regression," Journal of Classification, vol. 17, pp. 273-296, 2000.

[16] J. Isern-Fontanet, B. Chapron, G. Lapeyre and P. Klein, "Potential use of microwave sea surface temperatures for the estimation of ocean currents," Geophysical Research Letters, vol. 33, pp. L24608, 2006.

[17] J. Isern-Fontanet, G. Lapeyre, P. Klein, B. Chapron and M. W. Hecht, "Three-dimensional reconstruction of oceanic mesoscale currents from surface information," Journal of Geophysical Research, vol. 113, pp. C09005, 2008.

[18] J. B. Kadane and N. A. Lazar, "Methods and criteria for model selection," Journal of the American Statistical Association, vol. 99, pp. 279290, 2004.

[19] P. Klein, J. Isern-Fontanet, G. Lapeyre, G. Roullet, E. Danioux, B. Chapron, S. Le Gentil and H. Sasaki, "Diagnosis of vertical velocities in the upper ocean from high resolution sea surface height," Geophysical Research Letters, vol. 36, pp. L12603, 2009.

[20] J. H LaCasce and A. Mahadevan, "Estimating subsurface horizontal and vertical velocities from sea-surface temperature," Journal of Marine Research, vol. 64, pp. 695-721, 2006.

[21] G. Lapeyre and P. Klein, "Dynamics of the upper oceanic layers in terms of surface quasigeostrophy theory," Journal of Physical Oceanography, vol. 36, pp. 165-176, 2006.

[22] E. W. Leuliette and J. M. Whar, "Coupled pattern analysis of sea surface temperature and TOPEX/Poseidon sea surface height," Journal of Physical Oceanography, vol. 29, pp. 599-611, 1999.

[23] J. Marcello, F. Eugenio, F. Marqués, A. Hernandez-Guerra and A. Gasull, "Motion estimation techniques to automatically track oceanographic thermal structures in multisensor image sequences," Geoscience and Remote Sensing, IEEE Transactions on, vol. 46, pp. 2743-2762, 2008.

[24] M. Saraceno, C. Provost and A. R. Piola, "On the relationship between satellite-retrieved surface temperature fronts and chlorophyll a in the western South Atlantic," Journal of Geophysical Research: Oceans (19782012), vol. 110, pp. C11, 2005.

[25] R. Schlittgen, "A weighted least-squares approach to clusterwise regression," Advances in Statistical Analysis, vol. 95, pp. 205-217, 2011.

[26] X. Wu, X. H. Yan, Y. H. Jo and W. T. Liu, "Estimation of subsurface Temperature anomaly in the North Atlantic using a self-organizing map neural network," Journal of Atmospheric and Oceanic Technology, vol. 29, pp. 1675-1688, 2012.

[27] Y. $\mathrm{Xu}$ and $\mathrm{L}$. Fu, "The effects of altimeter instrument noise on the estimation of the wavenumber spectrum of the sea surface height," Journal of Physical Oceanography, in press. 
Dynamical mode 1

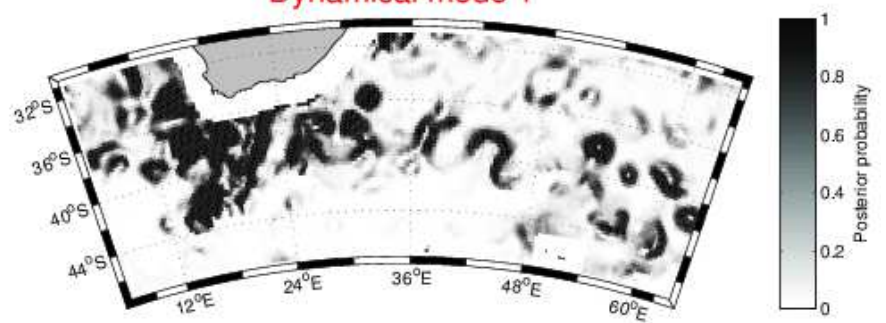

Dynamical mode 2
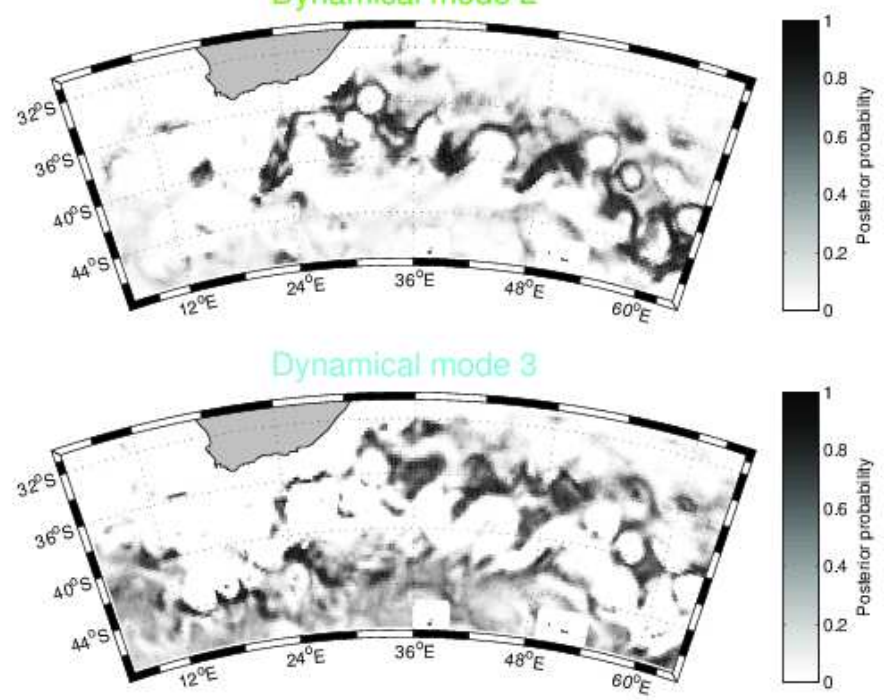

Dynamical mode 4

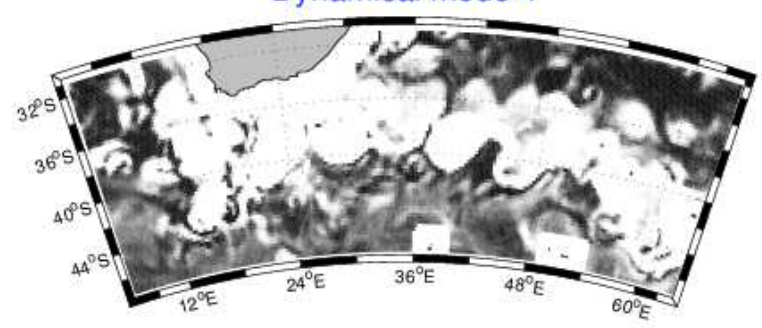

(a) $1^{\text {st }}$ of March, 2004

Dynamical mode 1

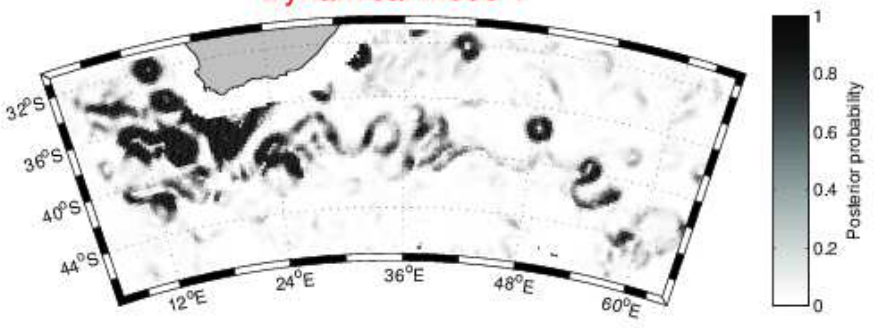

Dynamical mode 2
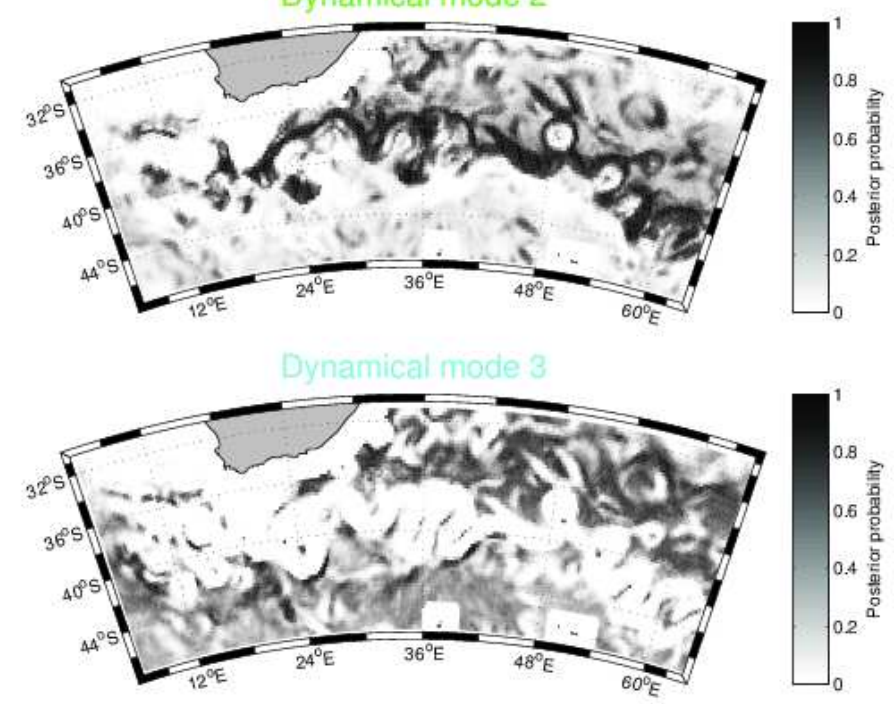

Dynamical mode 4

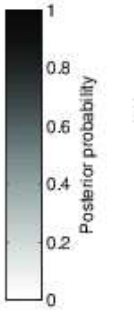

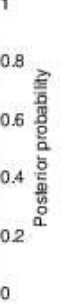

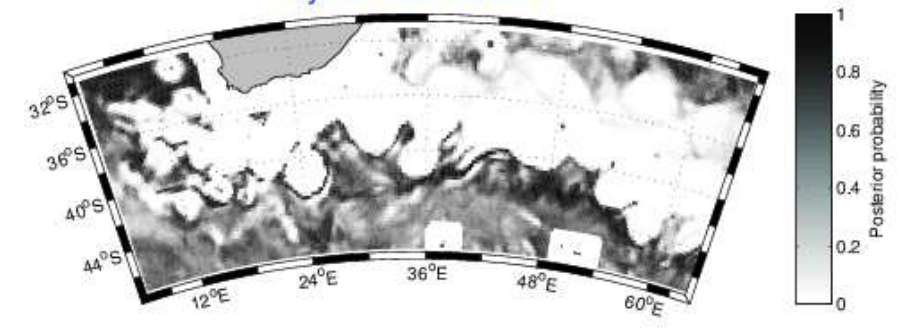

(b) $1^{\text {st }}$ of September, 2004

Fig. 4. Maps of the posterior probabilities given in Eq. (7) of the dynamical modes given the SST and SSH fields, the $1^{\text {st }}$ of March (a) and the $1^{\text {st }}$ of September (b), 2004 within the Agulhas current. We use a four-class latent regression model fitted from the whole year 2004 (see text for details). For a given location and time, the sum of the four probabilities is equal to 1 . The animations of the daily maps is available as supplementary material.

TABLE I

CORRELATION AND RMSE STATISTICS WITHIN THE AgUlHAS CURRENT FOR THE WHOLE YEAR 2004. THE LABELS "MADT", "LINEAR" AND “LATENT" REFER RESPECTIVELY TO THE TRUE MADT DATA, THE LINEAR MODEL AND THE PROPOSED LATENT CLASS REGRESSION MODEL. ALL THE CORRELATIONS ARE STATISTICALLY SIGNIFICANT (P-VALUES $<10^{-3}$ ).

\begin{tabular}{lc|cc}
\hline \multicolumn{2}{l}{ Correlation (RMSE) } & SSH & $(\mathrm{U}, \mathrm{V})$ \\
\hline MADT & LINEAR & $0.89(0.22)$ & $0.61(0.19)$ \\
MADT & LATENT & $0.96(0.16)$ & $0.72(0.16)$ \\
\hline
\end{tabular}

TABLE II

CORRELATION AND RMSE STATISTICS FOR SPATIO-TEMPORAL LOCATIONS ASSIGNED TO EACH DYNAMICAL MODE ACCORDING TO POSTERIOR PROBABILITIES COMPUTED IN EQ. (7) WITHIN THE AGULHAS CURRENT FOR THE WHOLE YEAR 2004. THE LABELS "MADT", "SQG" AND "LATENT" REFER RESPECTIVELY TO THE TRUE MADT DATA, A SURFACE QUASI-GEOSTROPHIC HYPOTHESIS AND THE PROPOSED LATENT CLASS REGRESSION MODEL. ALL THE CORRELATIONS ARE STATISTICALLY SIGNIFICANT (P-VALUES $<10^{-3}$ ).

\begin{tabular}{rl|cccc}
\hline \multicolumn{2}{c|}{ Correlation (RMSE) } & Mode 1 & Mode 2 & Mode 3 & Mode 4 \\
\hline SSH $_{\text {MADT }}$ & SSH & $0.72(0.26)$ & $0.93(0.16)$ & $0.97(0.13)$ & $0.96(0.12)$ \\
$(\mathrm{U}, \mathrm{V})_{\text {MADT }}$ & $(\mathrm{U}, \mathrm{V})_{\text {LATENT }}$ & $0.62(0.33)$ & $0.88(0.15)$ & $0.63(0.08)$ & $0.88(0.06)$ \\
$(\mathrm{U}, \mathrm{V})_{\text {MADT }}$ & $(\mathrm{U}, \mathrm{V})_{\text {SQG }}$ & $0.33(0.41)$ & $0.63(0.28)$ & $0.27(0.25)$ & $0.68(0.18)$ \\
\hline
\end{tabular}



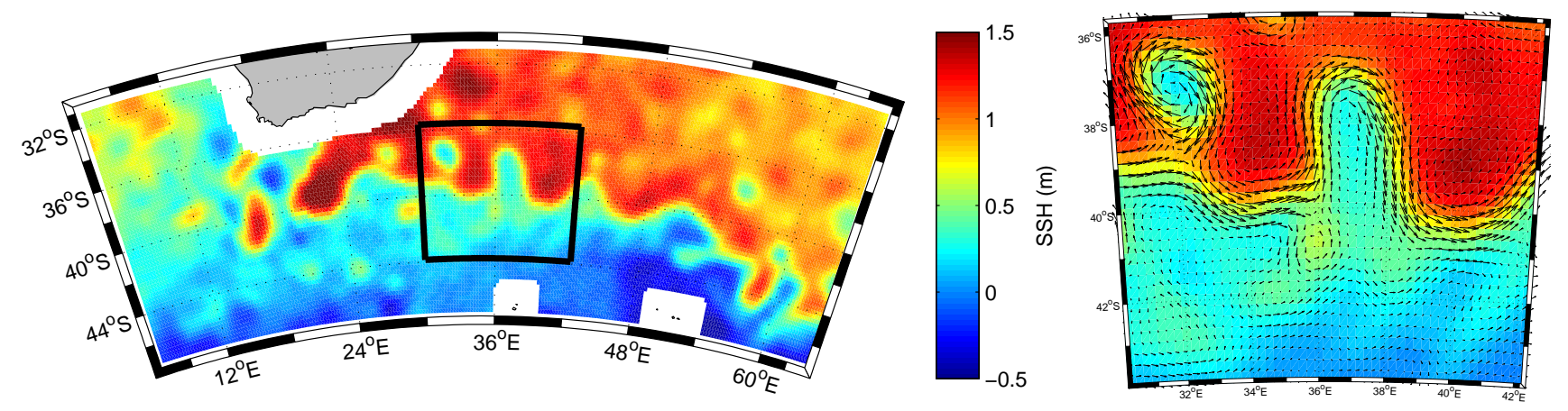

(a) True MADT data
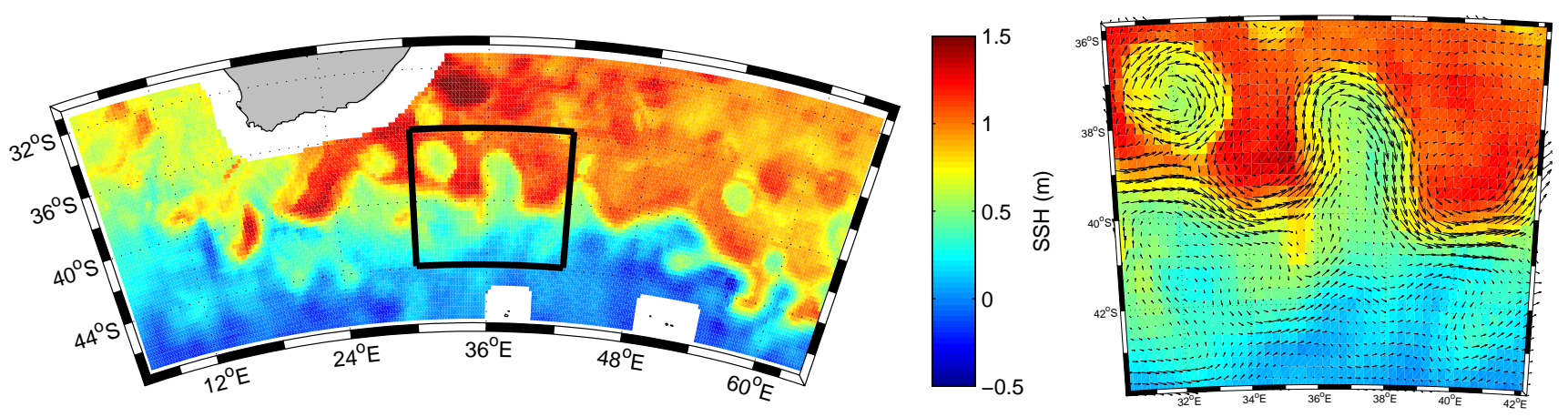

(b) Latent class regression
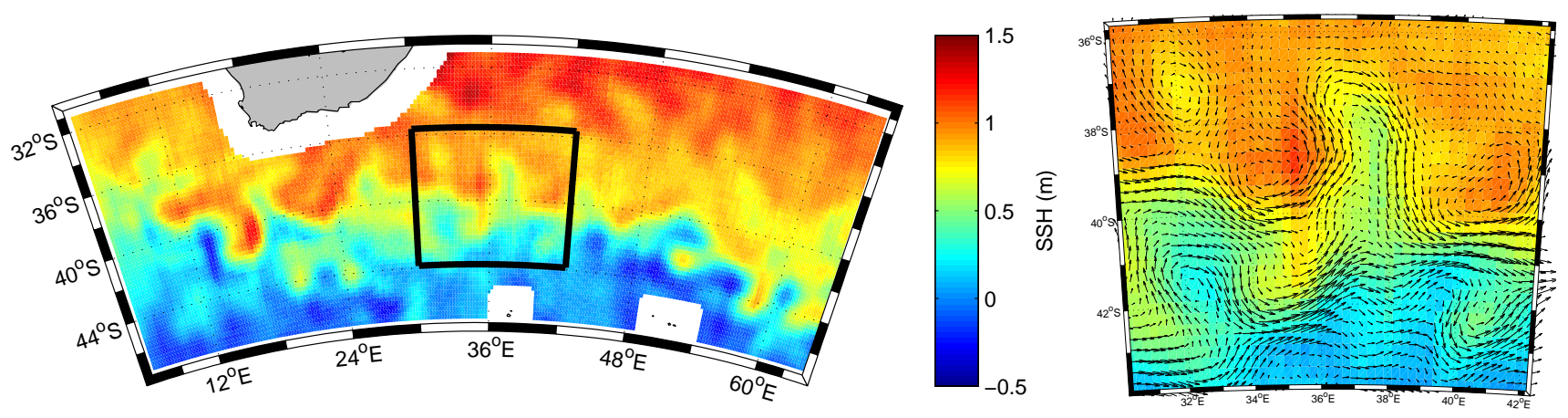

(c) Linear regression

Fig. 7. True Maps of Absolute Dynamic Topography (MADT) data (a) and the results for the proposed latent class regression model (b) using Eq. (10) and for the linear model (c), the $1^{\text {st }}$ of January, 2004 within the Agulhas current. The left column corresponds to the SSH and the right column corresponds to the SSH and surface currents $(\mathrm{U}, \mathrm{V})$ for the zone depicted in Fig. 1 and in the black box. 